\title{
Diagnostic Performances of Commercial ELISA, Indirect Hemagglutination, and Western Blot in Differentiation of Hepatic Echinococcal and Non-Echinococcal Lesions: A Retrospective Analysis of Data from a Single Referral Centre
}

\author{
Ambra Vola, ${ }^{1}$ Tommaso Manciulli, ${ }^{2}$ Annalisa De Silvestri, ${ }^{3}$ Raffaella Lissandrin,,${ }^{1,2}$ Mara Mariconti,,${ }^{1,2}$ Mar Siles-Lucas, ${ }^{4}$ \\ Enrico Brunetti, ${ }^{1,2}$ and Francesca Tamarozzi ${ }^{5 *}$ \\ ${ }^{1}$ Unit of Infectious and Tropical Diseases, San Matteo Hospital Foundation, Pavia, Italy; ${ }^{2}$ Department of Clinical, Surgical, Diagnostic and Pediatric \\ Sciences, University of Pavia, Pavia, Italy; ${ }^{3}$ Clinical Epidemiology and Biometric Unit, San Matteo Hospital Foundation, Pavia, Italy; ${ }^{4}$ Instituto de \\ Recursos Naturales y Agrobiología de Salamanca, Consejo Superior de Investigaciones Científicas, Salamanca, Spain; ${ }^{5}$ Department of Infectious \\ Diseases, WHO Collaborating Centre for the Epidemiology, Detection and Control of Cystic and Alveolar Echinococcosis, Istituto Superiore di \\ Sanità, Rome, Italy
}

\begin{abstract}
The diagnosis of cystic echinococcosis (CE) is based on imaging. Serology supports imaging in suspected cases, but no consensus exists on the algorithm to apply when imaging is inconclusive. We performed a retrospective analysis of serology results of patients with untreated hepatic CE and non-CE lesions, seen from 2005 to 2017, to evaluate their accuracy in the differential diagnosis of hepatic CE. Serology results of three seroassays for echinococcosis (ELISA RIDASCREEN, indirect hemagglutination (IHA) Cellognost, and Western blot LDBIO) and clinical characteristics of eligible patients were retrieved. Patients were grouped as having active or inactive CE and liquid or solid non-CE lesions. Sensitivity, specificity, and diagnostic accuracy were compared between scenarios encompassing different test combinations. Eligible patients included 104 patients with CE and 257 with non-CE lesions. Sensitivity and diagnostic accuracy of Western blot (WB) were significantly higher than those of the following: 1) IHA or ELISA alone, 2) IHA+ELISA interpreted as positive if both or either tests positive, and 3) IHA+ELISA confirmed by WB if discordant. The best performances were obtained when WB was applied on discordant or concordant negative IHA+ELISA. Analyses performed within "active CE $(n=52)$ versus liquid non-CE $(n=245)$ " and "inactive CE $(n=52)$ versus solid non-CE $(n=12)$ " groups showed similar results. Specificity was high for all tests $(0.99-1.00)$ and did not differ between test combination scenarios. WB may be the best test to apply in a one-test approach. Two first-level tests confirmed by WB seem to provide the best diagnostic accuracy. Further studies should be performed in different settings, especially where lower test specificity is likely.
\end{abstract}

\section{INTRODUCTION}

Cystic echinococcosis (CE) is a neglected parasitic zoonosis caused by the larval stage (metacestode) of the tapeworm Echinococcus granulosus sensu lato. The parasite is transmitted between canids, definitive hosts, and ungulate intermediate hosts. CE is unevenly distributed often over vast and underserved rural areas where livestock-breeding communities live. Humans are accidental intermediate hosts, acquiring the infection through the ingestion of infective parasitic eggs.

Echinococcal cysts may develop in humans in any organ or tissue, most commonly in the liver. Most infected people, especially those harboring abdominal $\mathrm{CE}$, are asymptomatic or paucisymptomatic, and often, echinococcal cysts are diagnosed during imaging examinations performed for other reasons. The diagnosis of $\mathrm{CE}$ is based on imaging, primarily ultrasound (US) for the abdominal locations. ${ }^{1} \mathrm{CE}$ cysts pass through different stages, as described in the WHO-IWGE (Informal Working Group on Echinococcosis) classification. ${ }^{1}$ The differential diagnosis of CE cysts may be broad, ranging from harmless biliary cysts to malignancies. Pathognomonic features of CE, when present, can be visualized on US; however, US is an operator-dependent examination and good-quality US machines and/or specific expertise on the recognition of CE characteristics are not widely available. Serology is used to support imaging in doubtful cases. However, presently available serology tests are not standardized, and their performances

* Address correspondence to Francesca Tamarozzi, Istituto Superiore di Sanità, Viale Regina Elena 299, Rome 00161, Italy. E-mail: francesca.tamarozzi@iss.it vary greatly, overall being quite unsatisfactory. ${ }^{2}$ Several factors, including CE cyst location, stage, size, number, and previous treatments, influence the outcome of serology tests. ${ }^{3}$ This induces a variable rate of false-negative results. False positivity also occurs because of cross-reactivity with other parasites or other possible mechanisms such as exposure to the parasite in endemic areas and nonspecific reactions. ${ }^{4}$ Consequently, the correct interpretation of serology is challenging.

Although some diagnostic algorithms have been proposed, ${ }^{5}$ at present, there is no consensus on the application and interpretation of serology when imaging is inconclusive; most studies investigating the performance of seroassays were not designed to assess their usefulness for the differential diagnosis of detected lesions. We performed a retrospective analysis of data on three commercially available serological tests applied to patients with untreated hepatic CE cysts and non-CE lesions, seen at the outpatient clinic of the Unit of Infectious and Tropical Diseases, San Matteo Hospital Foundation, Pavia, Italy, a referral center for CE located in a non-endemic area in northern Italy. The aim of this work was to evaluate the diagnostic accuracy of serology results in the differential diagnosis of hepatic CE.

\section{MATERIALS AND METHODS}

The study was a retrospective analysis of data from routine diagnostic tests. Databases of the CE clinic were searched, and clinical and serological data of eligible patients diagnosed between 2005 and 2017 were retrieved, a period when the same three serology tests were consistently used. The criteria for inclusion were as follows: 1) presence of only hepatic CE or 
suspect CE lesion(s) visualized on US, 2) having been tested with the same three serology assays (see the following text), and 3) not having received previous treatment for CE. All data were pseudonymized before analysis; ethical clearance was not required for this work.

Patients with CE were diagnosed and staged using US by experienced physicians. ${ }^{1}$ In some cases, the diagnosis of CE could be confirmed by observing a change in cyst morphology or seroconversion on albendazole administration or after observing the presence of protoscoleces in the cyst fluid following percutaneous puncture. Cystic echinococcosis stages CE1, CE2, CE3a, and CE3b (i.e., those cysts containing liquid components) were grouped as "active"; CE4 and CE5 cysts (i.e., with solid appearance) were grouped as "inactive." Patients with more than one cyst were classified as having active CE if at least one cyst was in stages CE1 to CE3b; patients were classified as having inactive CE if all cysts were in stage CE4 or CE5. Non-CE lesions were classified as "liquid non-CE" if they had cystic appearance with liquid components and as "solid non-CE" if no liquid component was observed on US. The diagnosis of these non-CE lesions was based on US, in some occasions aided by other investigations, including percutaneous aspiration, contrast-enhanced imaging, Doppler US, and followup after ex juvantibus albendazole intake, showing no changes in cyst morphology.

The three commercial serology assays were performed after the US examination. They were ELISA RIDASCREEN Echinococcus IgG (R-Biopharm, Darmstadt, Germany), indirect hemagglutination (IHA) Cellognost Echinococcosis (Siemens, Erlangen, Germany), and Western Blot Echinococcus WB IgG (LDBIO Diagnostics, Lyon, France). All tests were carried out as routine diagnostic procedures and were performed and interpreted according to the manufacturers' instructions.

Results of serology tests were analyzed qualitatively (positive/ negative). Sensitivity, specificity, positive and negative predictive values, positive and negative likelihood ratios, pretest probability, and positive and negative posttest probabilities, with 95\% confidence intervals $(\mathrm{Cl})$, were calculated for each test and their combinations, on the whole cohort and on separated groups ("active CE versus liquid non-CE" and "inactive CE versus solid non-CE" lesions). Sensitivity and specificity of different scenarios were compared using McNemar's test; diagnostic accuracies were analyzed by comparing the area under the curve (AUC) using a nonparametric approach for correlated receiver operating characteristic curves. ${ }^{6}$ Analyses were performed using the MedCalc Bayesian Analysis Model (www.medcalc.com) and Stata 15.1 (StataCorp 2017, College Station, TX).

\section{RESULTS}

One hundred four patients with hepatic $C E$ and 257 patients with non-CE lesions were included in the cohort. Cystic echinococcosis diagnosis in five patients with CE-suspected lesions was confirmed based on seroconversion $(n=3)$, observation of hooks in the aspirated fluid $(n=1)$, and progression from an active to inactive stage $(n=1)$. In 27 patients, a non-CE diagnosis was confirmed by percutaneous aspiration ( $n=16)$, contrast-enhanced imaging or Doppler US $(n=7)$, and ex juvantibus albendazole administration $(n=4)$. Non-CE lesions included biliary cysts, hemangiomas, neoplasms, hematomas, abscesses, focal steatosis, and a textiloma.
Clinical and serological characteristics of the cohort are summarized in Table 1. In the non-CE group, only three patients had positive serology for CE on IHA and/or ELISA. No patient with non-CE lesions was positive on WB.

We first analyzed the whole cohort and calculated the diagnostic performances of the three tests applied separately (scenario 1: IHA, 2: ELISA, and 3: WB) (Tables 2 and 3, Webonly Supplemental Table 1). The specificity of WB was higher than that of IHA and ELISA, but the differences were not statistically significant. However, sensitivity and diagnostic accuracy of WB were significantly higher than those of the other single tests (sensitivity of WB versus IHA, $P=0.012$; sensitivity of WB versus ELISA, $P=0.034$; and diagnostic accuracy of WB versus IHA versus ELISA, $P=0.015$ ).

Western blot might be the test of choice for the differential diagnosis of hepatic CE when a single-test approach is used. However, WB is expensive, requires specifically trained personnel for its interpretation, and is often used only as a confirmatory second-level test. We, therefore, calculated the diagnostic performances of different scenarios where either WB is not available or is used only as a confirmatory test (Tables 2 and 3, Web-only Supplemental Tables 2 and 3).

When we simulated the instance where IHA and ELISA were applied in parallel and interpreted as positive if concordant positive (scenario 4), sensitivities and diagnostic accuracies were significantly lower than those of either IHA or ELISA as single tests $(P=0.002$ and $P=0.003$, respectively). Western blot still had, however, significantly better sensitivity and diagnostic accuracy than IHA+ELISA $(P<0.001)$ (Tables 2 and 3, Web-only Supplemental Table 2). On the contrary, in the instance when IHA and ELISA were applied in parallel and interpreted as positive if either test was positive (scenario 5), the test combination had significantly higher sensitivity and diagnostic accuracy than the single tests $(P=0.003$ and $P=0.002$, respectively), whereas there was no significant difference with WB alone. Sensitivity and diagnostic accuracy were significantly higher for the scenario when IHA and ELISA were interpreted as positive if either test was positive (scenario 5) than those of the scenario when IHA and ELISA were interpreted as positive if concordant positive (scenario 4) $(P<$ 0.001; Tables 2 and 3, Web-only Supplemental Table 2).

When applied in case of IHA+ELISA discordant results, the addition of WB as a confirmatory test (scenario 6 versus scenario 4) had statistically higher sensitivity and diagnostic accuracy $(P<$ 0.001). However, WB alone was still significantly more sensitive and accurate than this combination test scenario (Tables 2 and 3 , Web-only Supplemental Table 3). Furthermore, sensitivity and diagnostic accuracy were significantly higher when WB was applied in case of IHA+ELISA discordant and concordant negative results (scenario 7 ) than when WB was applied in case of IHA+ELISA discordant results only (scenario 6$)(P=0.002$ for sensitivity and $P<0.001$ for accuracy), whereas there was no significant difference with WB alone (scenario 3) (Tables 2 and 3, Web-only Supplemental Table 3).

When we then applied the same analyses to the "active CE versus liquid non-CE" and "inactive CE versus solid non-CE" groups, overall the same results as the whole cohort applied to the "inactive CE versus solid non-CE" comparison, whereas only a subset of comparisons reached statistical significance in the "active CE versus liquid non-CE" group. Results are detailed in Tables 2 and 3 and Web-only Supplemental Tables 1-3. 
TABLE 1

Clinical and serological characteristics of the CE and non-CE cohorts

\begin{tabular}{lcrrr}
\hline Group stage & Number (\%) & Indirect hemagglutination, positive (\%) & ELISA, positive (\%) & Western blot, positive (\%) \\
\hline CE cohort & & & & \\
Active & $52(50.0)$ & $40(76.9)$ & $39(75.0)$ & $2(40.0)$ \\
CE1 & $5(4.8)$ & $3(60.0)$ & $7(70.0)$ & $44(84.6)$ \\
CE2 & $10(9.6)$ & $7(70.0)$ & $12(92.3)$ & $9(80.0)$ \\
CE3a & $13(12.5)$ & $11(84.6)$ & $18(75.0)$ & $10(76.0)$ \\
CE3b & $24(23.1)$ & $19(79.2)$ & $10(19.2)$ & $21(87.5)$ \\
Inactive & $52(50.0)$ & $10(19.2)$ & $9(32.1)$ & $16(30.8)$ \\
CE4 & $28(26.9)$ & $6(21.4)$ & $49(47.1)$ & $11(39.3)$ \\
CE5 & $24(23.1)$ & $4(16.7)$ & & $5(20.8)$ \\
Total & $104(100)$ & $50(48.1)$ & $2(0.8)$ & $60(57.7)$ \\
Non-CE Cohort & & & $0(0.0)$ & $0(0.0)$ \\
Liquid & $245(95.3)$ & $2(0.8)$ & $2(0.8)$ & $0(0.0)$ \\
Solid & $12(4.7)$ & $0(0.0)$ & & $0(0.0)$ \\
Total & $257(100)$ & $2(0.8)$ & &
\end{tabular}

For all these analyses performed on the whole cohort or on the "active CE versus liquid non-CE" and "inactive CE versus solid non-CE" groups separately, specificity did not differ significantly between scenarios.

\section{DISCUSSION}

The differential diagnosis of CE may be challenging. When imaging is inconclusive, serology plays a practical role in the diagnostic path; however, no consensus algorithm is available to guide the application and interpretation of serology. To our knowledge, this is the first study assessing the diagnostic accuracy of commercial seroassays for the differential diagnosis of hepatic CE, using a cohort of CE cases well characterized in terms of cyst stages and matching non-CE lesions, which were in the differential diagnosis of CE. One previous study investigated the diagnostic performances of variable combinations of three in-house tests for the diagnosis of liver CE. ${ }^{7}$ However, they used a heterogeneous cohort of CE cases without staging and patients with cholelithiasis, a condition not normally included in the differential diagnosis for hepatic CE, as controls. Although not comparable, the authors concluded that the best diagnostic accuracy was obtained using one or two first-line tests, followed by a highly specific third test, similarly to what can be derived from our data.

Our retrospective data show a surprisingly high specificity of all serology tests applied, with overall $1 \%$ false-positive test rate in patients with non-CE lesions. Explanation may be that

TABLE 2

Diagnostic performances of the three tests applied separately or in combination on the whole cohort and divided by groups

\begin{tabular}{|c|c|c|c|c|c|}
\hline Scenario & $\begin{array}{l}\text { Sensitivity } \\
(95 \% \mathrm{Cl})\end{array}$ & $\begin{array}{l}\text { Specificity } \\
(95 \% \text { Cl) }\end{array}$ & $\begin{array}{l}\text { Positive predictive value } \\
\qquad(95 \% \mathrm{Cl})\end{array}$ & $\begin{array}{l}\text { Negative predictive value } \\
(95 \% \mathrm{Cl})\end{array}$ & $\begin{array}{l}\text { Area under the curve } \\
(95 \% \mathrm{Cl})\end{array}$ \\
\hline \multicolumn{6}{|l|}{ Whole cohort } \\
\hline 1) IHA alone & $0.48(0.41-0.56)$ & $0.99(0.97-1.00)$ & $0.96(0.93-0.98)$ & $0.82(0.78-0.86)$ & $0.736(0.688-0.785)$ \\
\hline 2) ELISA alone & $0.47(0.42-0.52)$ & $0.99(0.97-1.00)$ & $0.96(0.93-0.98)$ & $0.82(0.78-0.86)$ & $0.732(0.683-0.780)$ \\
\hline 3) WB alone & $0.58(0.52-0.63)$ & $1.00(0.99-1.00)$ & $1.00(0.99-1.00)$ & $0.85(0.81-0.89)$ & $0.788(0.741-0.836)$ \\
\hline 4) IHA+ELISA & $0.38(0.33-0.44)$ & $0.99(0.98-1.00)$ & $0.98(0.95-0.99)$ & $0.80(0.75-0.84)$ & $0.690(0.643-0.737)$ \\
\hline 5) IHA and/or ELISA & $0.57(0.51-0.62)$ & $0.99(0.97-0.99)$ & $0.95(0.92-0.97)$ & $0.85(0.81-0.88)$ & $0.788(0.729-0.826)$ \\
\hline 6) $I H A+E L I S A$ and + WB if discordant & $0.50(0.45-0.55)$ & $0.99(0.98-1.00)$ & $0.98(0.96-0.99)$ & $0.83(0.79-0.87)$ & $0.748(0.700-0.796)$ \\
\hline $\begin{array}{l}\text { 7) IHA+ELISA and + WB if } \\
\text { discordant or negative }\end{array}$ & $0.59(0.54-0.65)$ & $0.99(0.98-1.00)$ & $0.98(0.96-0.99)$ & $0.86(0.82-0.89)$ & $0.796(0.749-0.844)$ \\
\hline \multicolumn{6}{|l|}{ Active CE vs. liquid non-CE } \\
\hline 1) IHA alone & $0.77(0.72-0.81)$ & $0.99(0.97-1.00)$ & $0.95(0.92-0.97)$ & $0.95(0.92-0.97)$ & $0.880(0.822-0.939)$ \\
\hline 2) ELISA alone & $0.75(0.69-0.80)$ & $0.99(0.97-1.00)$ & $0.95(0.92-0.97)$ & $0.95(0.92-0.97)$ & $0.871(0.811-0.931)$ \\
\hline 3) WB alone & $0.85(0.80-0.88)$ & $1.00(0.98-1.00)$ & $1.00(0.98-1.00)$ & $0.97(0.94-0.98)$ & $0.923(0.873-0.972)$ \\
\hline 4) IHA+ELISA & $0.71(0.66-0.76)$ & $0.99(0.98-1.00)$ & $0.97(0.95-0.99)$ & $0.94(0.91-0.96)$ & $0.854(0.791-0.916)$ \\
\hline 5) IHA and/or ELISA & $0.81(0.76-0.85)$ & $0.99(0.96-0.99)$ & $0.93(0.90-0.96)$ & $0.96(0.93-0.98)$ & $0.898(0.843-0.952)$ \\
\hline $\begin{array}{l}\text { 6) IHA+ELISA and +WB if } \\
\text { discordant }\end{array}$ & $0.81(0.76-0.85)$ & $0.99(0.98-1.00)$ & $0.98(0.95-0.99)$ & $0.96(0.93-0.98)$ & $0.902(0.847-0.956)$ \\
\hline 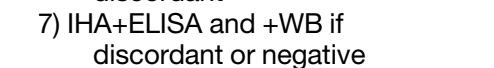 & $0.88(0.84-0.92)$ & $0.99(0.98-1.00)$ & $0.98(0.95-0.99)$ & $0.98(0.95-0.99)$ & $0.940(0.896-0.984)$ \\
\hline \multicolumn{6}{|l|}{ Inactive CE vs. solid non-CE } \\
\hline 1) IHA alone & $0.19(0.11-0.31)$ & $1.00(0.93-1.00)$ & $1.00(0.93-1.00)$ & $0.22(0.13-0.35)$ & $0.596(0.542-0.650)$ \\
\hline 2) ELISA alone & $0.19(0.11-0.31)$ & $1.00(0.93-1.00)$ & $1.00(0.93-1.00)$ & $0.22(0.13-0.35)$ & $0.596(0.542-0.650)$ \\
\hline 3) WB alone & $0.31(0.20-0.44)$ & $1.00(0.93-1.00)$ & $1.00(0.93-1.00)$ & $0.25(0.15-0.38)$ & $0.654(0.590-0.717)$ \\
\hline 4) IHA+ELISA & $0.06(0.02-0.15)$ & $1.00(0.93-1.00)$ & $1.00(0.93-1.00)$ & $0.20(0.11-0.32)$ & $0.529(0.497-0.561)$ \\
\hline 5) IHA and/or ELISA & $0.33(0.22-0.46)$ & $1.00(0.93-1.00)$ & $1.00(0.93-1.00)$ & $0.25(0.16-0.38)$ & $0.663(0.599-0.728)$ \\
\hline 6) $I H A+E L I S A$ and +WB if discordant & $0.19(0.11-0.31)$ & $1.00(0.93-1.00)$ & $1.00(0.93-1.00)$ & $0.22(0.13-0.35)$ & $0.596(0.542-0.650)$ \\
\hline 7) IHA+ELISA and +WB if discordant & $0.31(0.20-0.44)$ & $1.00(0.93-1.00)$ & $1.00(0.93-1.00)$ & $0.25(0.15-0.38)$ & $0.654(0.590-0.717)$ \\
\hline
\end{tabular}

$\mathrm{CE}=$ cystic echinococcosis; $\mathrm{IHA}=$ indirect hemagglutination; $\mathrm{WB}=$ Western blot. The complete set of diagnostic performance parameters, including positive and negative likelihood ratios and pre- and positive/negative posttest probabilities, is presented in the Web-only Supplemental Tables 1-3. 
TABLE 3

Statistical significance of the comparisons of diagnostic accuracies (AUC) of different scenarios of the three tests applied separately or in combination on the whole cohort and divided by groups

\begin{tabular}{|c|c|c|c|}
\hline \multirow[b]{2}{*}{ Compared scenarios } & \multicolumn{3}{|c|}{ Comparison of AUC $P$-value (superior scenario) } \\
\hline & Whole cohort & Active $C E$ vs. liquid non-CE & Inactive CE vs. solid non-CE \\
\hline 1) IHA vs. 2) ELISA vs. 3)WB & $0.015(3)$ & 0.171 & 0.081 \\
\hline 1) IHA vs. 4) IHA+ELISA & $0.002(1)$ & 0.103 & $0.005(1)$ \\
\hline 2) ELISA vs. 4) IHA+ELISA & $0.003(2)$ & 0.207 & $0.005(2)$ \\
\hline 3) WB vs. 4) IHA+ELISA & $<0.001(3)$ & $0.025(3)$ & $<0.001(3)$ \\
\hline 1) IHA vs. 5) IHA and/or ELISA & $0.003(5)$ & 0.207 & $0.005(5)$ \\
\hline 2) ELISA vs. 5) IHA and/or ELISA & $0.002(5)$ & 0.103 & $0.005(5)$ \\
\hline 3) WB vs. 5) IHA and/or ELISA & 0.618 & 0.289 & 0.783 \\
\hline 4) IHA+ELISA vs. 5) IHA and/or ELISA & $<0.001(5)$ & $0.035(5)$ & $<0.001(5)$ \\
\hline 3) WB vs. 6) IHA+ELISA; + WB if disc & $0.014(3)$ & 0.370 & $0.010(3)$ \\
\hline 4) IHA+ELISA vs. 6) IHA+ELISA; + WB if disc & $<0.001(6)$ & $0.020(6)$ & $0.005(6)$ \\
\hline $\begin{array}{l}\text { 3) WB vs. 7) IHA+ELISA; + WB if disc or } \\
\text { negative }\end{array}$ & 0.276 & 0.207 & 1.000 \\
\hline $\begin{array}{l}\text { 5) IHA and/or ELISA vs. 7) IHA+ELISA; + WB } \\
\text { if disc or negative }\end{array}$ & 0.361 & $0.024(7)$ & 0.783 \\
\hline $\begin{array}{l}\text { 6) IHA+ELISA; + WB if disc vs. 7) IHA+ELISA; + } \\
\text { WB if disc or negative }\end{array}$ & $<0.001(7)$ & $0.039(7)$ & $0.010(7)$ \\
\hline
\end{tabular}

AUC = area under the curve; CE = cystic echinococcosis; disc = discordant; IHA = indirect hemagglutination; WB = Western blot. Statistically significant differences are indicated in bold. The complete set of comparisons, including comparisons between sensitivities and specificities, is presented in the Web-only Supplemental Tables 1-3.

we assessed tests' specificity on a particular cohort of patients, defined by the presence of lesions that might enter differential diagnosis with hepatic CE. Using this type of control population is sensible because serology is applied as a complementary test after the visualization on imaging of a lesion suggestive of CE. Similar to our results here, when we used the same type of control cohort to investigate the diagnostic accuracy of commercial rapid diagnostic tests, specificities were generally higher than those obtained in studies assessing the accuracy of seroassays using other control groups. ${ }^{8}$ It must be noted, however, that our control cohort did not include any patients with alveolar echinococcosis $(A E)$ caused by E. multilocularis because of the absence of patients with this serious condition cared for in our hospital. This is a limitation of our study as AE is among the most important differential diagnoses of CE in co-endemic areas, and even the most species-specific serological tests show a high rate of cross-reactivity between the two species. ${ }^{9,10}$

Our results indicate that WB may be the best single test to apply for the differential diagnosis of hepatic CE, when a onetest approach is chosen. Western blot alone was still generally superior to the application of two different tests. When we explored the scenario where WB was not available and two first-level tests were used in parallel, we found the rather counter-intuitive result that overall diagnostic accuracy was significantly higher when only one of the first-level tests was applied or when the two tests were used together but a truepositive result was considered if just one of the two tests resulted positive. These findings were derived from the high specificity of all tests observed in our study and should be interpreted with caution as this high specificity would not necessarily apply in areas where potentially cross-reacting conditions may occur more frequently. Indeed, although not statistically significant, scenario 4 (IHA and ELISA applied in parallel and interpreted as positive if concordant positive), intuitively, showed higher specificity than scenarios 1 (IHA alone), 2 (ELISA alone), and 5 (IHA and ELISA applied in parallel and interpreted as positive if at least one test was positive). Especially in settings where cross-reactivity may be frequent, the choice of favoring sensitivity over specificity should be evaluated carefully for its repercussions on clinical management. When feasible, the application of two first-level tests, confirmed by WB in case of discordancy or concordant negative results, seems to provide the best diagnostic accuracy.

When examining the "active CE versus liquid non-CE" and "inactive CE versus solid non-CE" groups separately, overall results generally overlapped those of the whole cohort. The best diagnostic accuracy of the investigated serology tests (and their combinations) was found when serology was applied in the presence of active CE or non-CE lesions with "liquid content." This reflects the well-known higher sensitivity of serology tests in the presence of active and transitional cysts compared with inactive cysts. ${ }^{3,4}$ Taken as a whole, our results support the view that positive serology may be used to confirm a diagnosis of CE in the presence of reasonably high pretest probability, whereas a negative test, even if obtained by WB, cannot exclude CE. The very low negative posttest probability observed in the "active CE versus liquid non-CE" group might suggest the possibility to interpret a negative serology as a CE exclusion criterion in this group. However, this approach should be taken with caution and in light of costbenefit considerations, particularly whether it would be preferable risking to wrongly exclude a diagnosis of CE when serology is negative or to continue the diagnostic workup despite the low likelihood for the lesion to be CE. Besides the already mentioned absence of $\mathrm{AE}$ among the control conditions, this study has several limitations. First is the retrospective design, which is burdened by intrinsic selection bias. It was difficult in some cases, on the basis of clinical records, to disentangle whether serology results were taken into account or not in the final definition of CE versus non-CE case. Whenever possible, additional evidence confirming the diagnosis was sought. Also, no information was available on chest X-ray of patients with non-CE hepatic lesions and serology-positive results, to exclude that seropositivity derived from an extra-abdominal CE. Finally, our results cannot be generalized: patients had exclusively hepatic lesions, had never been treated for $\mathrm{CE}$, and were seen in a reference center located in a non-endemic area and with uncommon experience in the diagnosis of CE. Indeed, as tests' predictive values 
depend also on pretest probability, very different positive predictive value and negative predictive value may result from the application of the same tests not only in different geographical areas, but also to lesions more or less likely to be CE on imaging, in turn depending on the experience of the operator.

Conclusions of the study are as follows: 1) WB may the best single test to apply when a one-test approach is chosen; 2) the application of two first-level tests confirmed by WB in case of discordancy and concordant negative results seems to provide the best diagnostic accuracy; and 3) serology should not be used to exclude CE in the presence of a compatible lesion. Further studies are needed to evaluate the performance of tests in different settings, with different expertise, type, and ratio of $C E$ versus non-CE cases and using a prospective, diagnostic benefit design. Presently available commercial and in-house test, as well as new tests under development (recently reviewed in ref. 2), should be validated using an image-corresponding control group to provide information applicable by clinicians in their settings.

Received July 26, 2019. Accepted for publication September 10, 2019.

Published online October 28, 2019.

Note: Supplemental tables appear at www.ajtmh.org.

Acknowledgments: We thank Sam Goblirsch for editing the manuscript for language.

Financial support: This work was funded by the European Union Seventh Framework Program (FP7/2007-2013) under the project HERACLES, grant agreement no. 602051-to E. B.

Authors' addresses: Ambra Vola, Unit of Infectious and Tropical Diseases, San Matteo Hospital Foundation, Pavia, Italy, E-mail: ambra.vola@ gmail.com. Tommaso Manciulli, Department of Clinical, Surgical, Diagnostic and Pediatric Sciences, University of Pavia, Pavia, Italy, E-mail: tommaso.manciulli01@universitadipavia.it. Annalisa De Silvestri, Clinical Epidemiology and Biometric Unit, San Matteo Hospital Foundation, Pavia, Italy, E-mail: a.desilvestri@smatteo.pv.it. Raffaella Lissandrin, Mara Mariconti, and Enrico Brunetti, Unit of Infectious and Tropical Diseases, San Matteo Hospital Foundation, Pavia, Italy, and Department of Clinical, Surgical, Diagnostic and Pediatric Sciences, University of Pavia, Pavia, Italy, E-mails: raffaella.lissandrin@unipv.it, maramariconti@libero.it, and enrico.brunetti@unipv.it. Mar Siles-Lucas, Instituto de Recursos Naturales y Agrobiología de Salamanca, CSIC, Salamanca, Spain, E-mail: mmar.siles@irnasa.csic.es. Francesca Tamarozzi, Department of Infectious Diseases, WHO Collaborating Centre for the Epidemiology, Detection and Control of Cystic and Alveolar Echinococcosis, Istituto Superiore di Sanità, Rome, Italy, E-mail: francesca.tamarozzi@iss.it.

\section{REFERENCES}

1. Brunetti E, Kern P, Vuitton DA; Writing Panel for the WHO-IWGE, 2010. Expert consensus for the diagnosis and treatment of cystic and alveolar echinococcosis in humans. Acta Trop 114: $1-16$.

2. Siles-Lucas M, Casulli A, Conraths FJ, Müller N, 2017. Laboratory diagnosis of Echinococcus spp. in human patients and infected animals. Adv Parasitol 96: 159-257.

3. Lissandrin R, Tamarozzi F, Piccoli L, Tinelli C, De Silvestri A, Mariconti M, Meroni V, Genco F, Brunetti E, 2016. Factors influencing the serological response in hepatic Echinococcus granulosus infection. Am J Trop Med Hyg 94: 166-171.

4. Hernández-González A, Muro A, Barrera I, Ramos G, Orduña A, Siles-Lucas M, 2008. Usefulness of four different Echinococcus granulosus recombinant antigens for serodiagnosis of unilocular hydatid disease (UHD) and postsurgical follow-up of patients treated for UHD. Clin Vaccine Immunol 15: 147-153.

5. Siles-Lucas MM, Gottstein BB, 2001. Molecular tools for the diagnosis of cystic and alveolar echinococcosis. Trop Med Int Health 6: 463-475.

6. DeLong ER, DeLong DM, Clarke-Pearson DL, 1988. Comparing the areas under two or more correlated receiver operating characteristic curves: a nonparametric approach. Biometrics 44: 837-845

7. Manterola C, Cuadra A, Muñoz S, Sanhueza A, Bustos L, Vial M, Fonseca $F, 2005$. In a diagnostic test study the validity of three serodiagnostic test was compared in patients with liver echinococcosis. J Clin Epidemiol 58: 401-406.

8. Tamarozzi $\mathrm{F}$ et al., 2016. Comparison of the diagnostic accuracy of three rapid tests for the serodiagnosis of hepatic cystic echinococcosis in humans. PLoS Negl Trop Dis 10: e0004444.

9. Liance $M$, Janin V, Bresson-Hadni S, Vuitton DA, Houin R, Piarroux R, 2000. Immunodiagnosis of Echinococcus infections: confirmatory testing and species differentiation by a new commercial western blot. J Clin Microbiol 38: 3718-3721.

10. Knapp J, Sako Y, Grenouillet F, Bresson-Hadni S, Richou C, Gbaguidi-Haore H, Ito A, Millon L, 2014. Comparison of the serological tests ICT and ELISA for the diagnosis of alveolar echinococcosis in France. Parasite 21: 34. 